\title{
Catalogue of H-alpha emission stars in the Northern Milky Way
}

\author{
L. Kohoutek and R. Wehmeyer \\ Hamburger Sterwarte Gojenbergsweg 112, D-21029 Hamburg, Germany \\ Received July 30; accepted August 13, 1998
}

\begin{abstract}
The "Catalogue of Stars in the Northern Milky Way Having H-alpha in Emission" appears in Abhandlungen aus der Hamburger Sternwarte, Band $\mathrm{XI}$ in the year 1997. It contains 4174 stars, range $32^{\circ} \leq l^{\mathrm{II}}<214^{\circ},-10^{\circ}<b^{\mathrm{II}}<+10^{\circ}$ having the $\mathrm{H} \alpha$ line in emission. HBH stars and stars of further 99 lists taken from the literature till the end of 1994 were included in the catalogue. We give the cross-identification of stars from all lists used. The catalogue is also available in the Centre de Données, Strasbourg ${ }^{1}$ and at the Hamburg Observatory via internet.
\end{abstract}

Key words: catalogues - galaxy: general — stars: emission-line

We would like to announce the "Catalogue of Stars in the Northern Milky Way Having H-alpha in Emission" which appears in Abhandlungen aus der Hamburger Sternwarte, Band XI in the year 1997 in two parts: Part 1 - Catalogue, Part 2 - Charts. The catalogue contains 4174 stars in the Northern Milky Way, range $32^{\circ} \leq l^{\mathrm{II}}<214^{\circ},-10^{\circ}<b^{\mathrm{II}}<+10^{\circ}$ having the $\mathrm{H} \alpha$ line in emission and having been listed up till the end of 1994 .

The Schmidt camera $(80 / 120 \mathrm{~cm}, f=240 \mathrm{~cm}$, $4^{\circ}$-Prism, dispersion $1590 \mathrm{~A} / \mathrm{mm}$ at $\mathrm{H} \alpha$ ) of the Hamburg Observatory in Bergedorf was used. The plates, Kodak 103a-E + Schott RG1, exp. 60 min, widening 10" in RA, were taken by one author (L.K.) in the years 1964-1970. They were taken in 166 fields along the galactic equator, in RA between $18^{\mathrm{h}} 58^{\mathrm{m}} 5$ and $7^{\mathrm{h}} 02^{\mathrm{m}} 0$ and in Decl. between $-2.5^{\circ}$ and $+72.5^{\circ}$. The list of HBH stars (HamburgBergedorf stars having $\mathrm{H} \alpha$ in emission) is the product of a visual inspection of these spectral plates.

Within the framework of the programme "Hamburg Schmidt-camera Survey of faint Planetary Nebulae" numerous planetary nebulae were classified (K3-, K4-).

Send offprint requests to: L. Kohoutek

${ }^{1}$ ftp 130.79.128.5 or http://cdsweb.u-strasbg.fr
Unfortunately, no spectra in the blue region were available, so that many of these objects turned out to be not planetary nebulae but mainly various $\mathrm{H} \alpha$ emission-line stars.

Altogether there are $1979 \mathrm{HBH}$ stars, 1570 of which were already known and identical with the stars given in other lists (Table 1 of the catalogue), and 409 are new $\mathrm{H} \alpha$ emisssion line stars. We give the identification of $\mathrm{HBH}$ stars with altogether 99 lists. Besides 91 short lists there are the following eight main lists:

HD Henry Draper Catalogue or its Extensions; BD Bonner Durchmusterung;

$\mathrm{MWC}+\mathrm{AS}$ Mount Wilson Catalogue and Additional

Stars (Burwell et al. 1933-51);

LS Luminous Stars in the Northern Milky

Way (Hardorp et al. 1959-65);

BID Bidelman (from the year 1954);

HE3 Henize (from the year 1976);

TON Tonantzintla and Tacubaya Observatory (Chavira et al. 1953-56);

VES Vatican Observatory

(Corbally et al. 1977-83).

The cross-identification between all lists of $\mathrm{H} \alpha$ emission line stars is given, too.

There are 11 lists which were omitted for various reasons (Table 2). We were not able to find 18 stars having the $\mathrm{H} \alpha$ line in emission (Table 3 ).

The object list and the remarks contain the following columns:

Left side (A):

Running line numbers of stars;

Equatorial coordinates (equ. 2000.0) given in four accuracy categories: in $\mathrm{RA} \mathrm{a)} \pm 0.01 \mathrm{~s}, \mathrm{~b}) \pm 0.1 \mathrm{~s}, \mathrm{c}) \pm 1 \mathrm{~s}$, d) $\pm 0.1 \mathrm{~m}$;

Galactic coordinates;

Brightnesses and colour systems given in the literature; Spectra given in the literature;

POSS O-charts on which the rectangular coordinates have been measured; 
HBH stars;

Classifications of the spectra of $\mathrm{HBH}$ stars;

Existence of finding charts (paper version) or plate numbers on which the finding chart is given (electronic version).

Right side (B):

Running line numbers of stars;

Names of the stars (names of the variables);

Star numbers in HD, BD, MWC+AS, LS, BID, HE3, TON, VES;

Star numbers in further 91 lists of stars having the $\mathrm{H} \alpha$ line in emission;

Existence of remarks.

Remarks (C):

Further discovery numbers, information on brightnesses and spectra, general information on the stars.

The finding charts were generally reproduced from the blue (O) prints of the National Geographic Society Palomar Observatory Sky Survey. The size of the majority of the charts is 7 arcmin square (scale about $1 \mathrm{~mm}=$ 12 arcsec). We present the finding charts of 2307 objects: of all $\mathrm{HBH}$ stars except of those which are BD stars, and of some stars from other lists for convenience. The finding charts are given in order of RA (2000.0).

In the elementary statistics we give at first the distribution of the stars on the celestial sphere divided into early-type and late-type stars. There are 339 stars in or near 51 clusters in our region. Altogether 2316 stars have a sufficiently accurate determination of the position, the authors together with collaborators determined or improved about 1550 positions of objects. Altogether 3654 stars in the catalogue $(88 \%)$ are indicated with brightness, 482 of which are variables. The variable stars will be treated later in a separate publication. Only 2351 stars (56\%) are indicated with spectral type.

We would like to point out the existing gaps in the material, not-measured brightnesses or missing spectral classifications.

The catalogue is also available in the Centre de Données, Strasbourg under the designation KW97. It is accessible using: either

ftp://cdsarc.u-strasbg.fr/pub/cats/III/205 or

http://vizier.u-strasbg.fr/cgi-bin/VizieR?-source= III $/ 205$

and also from the Hamburg Observatory via homepage (Kohoutek):

http://staix5.hs.uni-hamburg.de/german/persons/

kohoutek.html

\section{References}

Kohoutek L., Wehmeyer R., 1997, Catalogue of Stars in the Northern Milky Way Having H-alpha in Emission, Abhandl. Hamb. Sternw. XI

\section{Erratum}

In the paper version of the above $\mathrm{H}$-alpha Catalogue, Part 2 (Charts), Plate 25, 14th chart, instead of 4214-05 should be read 4214-15.

The electronic version (CDS, Hamburg Observatory homepage) is already corrected. 